\title{
Granularity, Multi-valued Logic, Bayes' Theorem and Rough Sets
}

\author{
Zdzisław Pawlak \\ Institute for Theoretical and Applied Informatics \\ Polish Academy of Sciences \\ ul. Bałtycka 5, 44000 Gliwice, Poland \\ e-mail:zpw@ii.pw.edu.pl
}

\begin{abstract}
Granularity of knowledge attracted attention of many researchers recently. This paper concerns this issue from the rough set perspective. Granularity is inherently connected with foundation of rough set theory. The concept of the rough set hinges on classification of objects of interest into similarity classes, which form elementary building blocks (atoms, granules) of knowledge. These granules are employed to define basic concepts of the theory. In the paper basic concepts of rough set theory will be defined and their granular structure will be pointed out. Next the consequences of granularity of knowledge for reasoning about imprecise concepts will be discussed. In particular the relationship between some ideas of Łukasiewicz's multi-valued logic, Bayes' Theorem and rough sets will be pointed out.
\end{abstract}

\section{Introduction}

This paper is an extended version of [15].

Information (knowledge) granulation, discussed recently by Prof. Zadeh $[27,28,29]$ seems to be a very important issue for computing science, logic, philosophy and others.

In this note we are going to discuss some problems connected with granularity of knowledge in the context of rough sets. First, discussion of granulation of knowledge in connection with rough and fuzzy sets has been presented by Dubois and Prade in [8]. Recently, an interesting study of information granulation in the framework of rough sets can be found in Polkowski and Skowron [16] and Skowron and Stepaniuk [20].

In rough set theory we assume that with every object some information is associated, and objects can be "seen" through the accessible information only. Hence, object with the same information cannot be discerned and appear as the same. This results in, that indiscernible objects of the universe form clusters of indistinguishable objects (granules, atoms, etc.). Thus from the rough set view the granularity of knowledge is due to the indiscernibility of objects caused by lack of sufficient information about them. Consequently granularity and indiscerniblity are strictly connected and the concept of indiscernibility seems to be prior to granularity. 
Current state of rough set theory and its application can be found in [19].

Indiscernibility attracted attention of philosophers for a long time and its first formulation can be attributed to Leibniz (cf. Forrest [9]), and is known as the principle of "the identity of indiscernibles". The principle says that not two objects have exactly the same properties, or in other words if all properties of objects $x$ and $y$ are the same then $x$ and $y$ are identical.

But what are "properties of objects"? and what does it mean "all properties"? A lot of philosophical discussions have been devoted to answer these questions (cf. e.g., Black [3], Forrest [9]), but we will refrain here from philosophical debate. Let us observe only that Leibniz approach to indiscernibility identifies indiscernibility with identity. The later is obviously an equivalence relation, i.e., it leads to partition of the universe into equivalence classes (granules) of objects which are indistinguishable in view of the assumed properties. Thus in the rough set approach granulation is a consequence of the Leibniz principle.

It is worthwhile to mention that indiscernibility can be also viewed in a wider context, as pointed out by Williamson [25]: "Intelligent life requires the ability discriminate, but not with unlimited precision". This is a very interesting issue however it lays outside the scope of this paper.

In rough set theory we assume empiristic approach, i.e., we suppose that properties are simply empirical data which can be obtained as a result of measurements, observations, computations, etc. and are expressed by values of a fixed, finite set of attributes, e.g., properties are attribute-value pairs, like (size, small), (color, red) etc. The idea could be also expressed in more general terms assuming as a starting point not a set of specific attributes but abstract equivalence relation, however, the assumed approach seems more intuitive.

Equivalence relation is the simplest formalization of the indiscernibility relation and is sufficient for many applications. However, more interesting seems to assume that the indiscernibility relation is formalized as a tolerance relation, i.e., transitivity of indiscernibilty is denied in this case, for, if $x$ is indiscernible from $y$ and $y$ is indiscernible from $z$ then not necessarily $x$ is indiscernible from $z$. Many authors have proposed tolerance relation as a basis for rough set theory (cf. e.g., Skowron and Stepaniuk [19]). This causes, however, some mathematical complications as well philosophical questions, because it leads to vague granules, i.e., granules without sharp boundaries, closely related to the boundary-line approach to vagueness (cf. e.g., Chattebrjee [7], Sorensen [22]).

Besides, instead of tolerance relation also more sophisticated mathematical models of indiscernibility, as a basis for rough set theory, have been proposed (cf. e.g., Krawiec, Slowinski, and Vanderpooten [11], Yao and Wong, [26], Ziarko [30]). Interested readers are advised to consult the mentioned above references, but for the sake of simplicity we will adhere in this paper 
to the equivalence relation as a mathematical formalization of the indiscernibility relation.

Since granules of knowledge can be considered as a basic building blocks of knowledge about the universe it seems that natural mathematical model for granulated knowledge can be based on ideas similar to that used in mereology proposed by Leśniewski [12], in which part of is the basic relation of this theory. Axioms of mereology, in particular in a version proposed by Suppes [23], seem to be natural candidate for this purpose. Moreover, rough mereology, extension of classical mereology proposed by Polkowski and Skowron in $[17,18]$, seems to be exceptionally suited to analyze granules of knowledge with not sharp boundaries (cf. Polkowski and Skowron [16], Skowron and Stepaniuk [20]).

It also worthwhile to mention in this context that granularity of knowledge has been also pursued in quantum physics. Its relation to fuzzy sets and rough sets has been first mentioned by Cattaneo $[5,6]$.

Recently a very interesting study of rough sets, granularity and foundations of mathematics and physics has been done by Apostoli and Kanda $[2]$.

Besides, it is also interesting to observe that computations and measurements are very good examples of granularity of information, for they are based in fact not on real numbers but on intervals, determined by the accuracy of computation or measurement.

\section{Basic Philosophy of Rough Sets}

The rough set philosophy is founded on the assumption that with every object of the universe of discourse we associate some information (data, knowledge). E.g., if objects are patients suffering from a certain disease, symptoms of the disease form information about patients. Objects characterized by the same information are indiscernible (similar) in view of the available information about them. The indiscernibility relation generated in this way is the mathematical basis of rough set theory.

Any set of all indiscernible (similar) objects is called an elementary concepts, and forms a basic granule (atom) of knowledge about the universe. Any union of some elementary concepts is referred to as crisp (precise) concept otherwise the set is rough (imprecise, vague).

Consequently each rough concept has boundary-line cases, i.e., objects which cannot be with certainty classified neither as members of the concept nor of its complement. Obviously crisp concepts have no boundary-line elements at all. That means that boundary-line cases cannot be properly classified by employing the available knowledge.

Thus, the assumption that objects can be "seen" only through the information available about them leads to the view that knowledge has granular structure. As a consequence vague concepts, in contrast to precise concepts, 
cannot be characterized in terms of elementary concepts. Therefore in the proposed approach we assume that any vague concept is replaced by a pair of precise concepts - called the lower and the upper approximation of the vague concept. The lower approximation consists of all elementary concepts which surely are included in the concept and the upper approximation contains all elementary concepts which possibly are included in the concept. Obviously, the difference between the upper and the lower approximation constitutes the boundary region of the vague concept. Approximations are two basic operations in rough set theory.

\section{Indiscernibility and Granularity}

As mentioned in the introduction, the starting point of rough set theory is the indiscernibility relation, generated by information about objects of interest. The indiscernibility relation is intended to express the fact that due to the lack of knowledge we are unable to discern some objects employing the available information. That means that, in general, we are unable to deal with single objects but we have to consider clusters of indiscernible objects, as fundamental concepts of knowledge.

Now we present above considerations more formally.

Suppose we are given two finite, non-empty sets $U$ and $A$, where $U$ is the universe, and $A$ - a set attributes. With every attribute $a \in A$ we associate a set $V_{a}$, of its values, called the domain of $a$. The pair $S=(U, A)$ will be called an information system. Any subset $B$ of $A$ determines a binary relation $I_{B}$ on $U$, which will be called an indiscernibility relation, and is defined as follows:

$x I_{B} y$ if and only if $a(x)=a(y)$ for every $a \in A$,

where $a(x)$ denotes the value of attribute $a$ for element $x$.

Obviously $I_{B}$ is an equivalence relation. The family of all equivalence classes of $I_{B}$, i.e., the partition determined by $B$, will be denoted by $U / I_{B}$, or simply $U / B$; an equivalence class of $I_{B}$, i.e., the block of the partition $U / B$, containing $x$ will be denoted by $B(x)$.

If $(x, y)$ belongs to $I_{B}$ we will say that $x$ and $y$ are $B$-indiscernible. Equivalence classes of the relation $I_{B}$ (or blocks of the partition $U / B$ ) are referred to as B-elementary concepts or B-granules.

In the rough set approach the elementary concepts are the basic building blocks (concepts) of our knowledge about reality.

\section{Approximations and Granularity}

Now the indiscernibility relation will be used to define basic operations in rough set theory, which are defined as follows:

$$
B_{*}(X)=\bigcup_{x \in U}\{B(x): B(x) \subseteq X\},
$$




$$
B^{*}(X)=\bigcup_{x \in U}\{B(x): B(x) \cap X \neq \emptyset\},
$$

assigning to every $X \subseteq U$ two sets $B_{*}(X)$ and $B^{*}(X)$ called the $B$-lower and the $B$-upper approximation of $X$, respectively.

Hence, the $B$-lower approximation of a concept is the union of all $B$ granules that are included in the concept, whereas the $B$-upper approximation of a concept is the union of all $B$-granules that have a nonempty intersection with the concept. The set

$$
B N_{B}(X)=B^{*}(X)-B_{*}(X)
$$

will be referred to as the B-boundary region of $X$.

If the boundary region of $X$ is the empty set, i.e., $B N_{B}(X)=\emptyset$, then $X$ is crisp (exact) with respect to $B$; in the opposite case, i.e., if $B N_{B}(X) \neq \emptyset$, $X$ is referred to as rough (inexact) with respect to $B$.

Rough sets can be also defined using a rough membership function, defined as

$$
\mu_{X}^{B}(x)=\frac{\operatorname{card}(B(x) \cap X)}{\operatorname{card}(B(x))} .
$$

Obviously

$$
\mu_{X}^{B}(x) \in[0,1] .
$$

Value of the membership function $\mu_{X}^{B}(x)$ is kind of conditional probability, and can be interpreted as a degree of certainty to which $x$ belongs to $X$ (or $1-\mu_{X}^{B}(x)$, as a degree of uncertainty).

The rough membership function, can be used to define approximations and the boundary region of a set, as shown below:

$$
\begin{gathered}
B_{*}(X)=\left\{x \in U: \mu_{X}^{B}(x)=1\right\}, \\
B^{*}(X)=\left\{x \in U: \mu_{X}^{B}(x)>0\right\}, \\
B N_{B}(X)=\left\{x \in U: 0<\mu_{X}^{B}(x)<1\right\} .
\end{gathered}
$$

The rough membership function can be generalized as follows (cf. Polkowski and Skowron [17]):

$$
\mu(X, Y)=\frac{\operatorname{card}(X \cap Y)}{\operatorname{card} X}
$$

where $X, Y \subseteq U, X \neq \emptyset$ and $\mu(\Phi, Y)=1$.

Function $\mu(X, Y)$ is an example of a rough inclusion [14] and expresses the degree to which $X$ is included in $Y$. Obviously, if $\mu(X, Y)=1$, then $X \subseteq Y$.

If $X$ is included in a degree $k$ we will write $X \subseteq_{k} Y$.

The rough inclusion function can be interpreted as a generalization of the mereological relation "part of", and reads as "part in a degree". 
Employing now the rough inclusion function we can represent approximations in an uniform way:

$$
\begin{aligned}
& B_{*}(X)=\bigcup_{x \in U}\{B(x): \mu(B(x), X)=1\}, \\
& B^{*}(X)=\bigcup_{x \in U}\{B(x): \mu(B(x), X)>0\} .
\end{aligned}
$$

Hence, the $B$-lower approximation of $X$ consists of all $B$-granules included in $X$, whereas the $B$-upper approximation of $X$ consists of all roughly included $B$-granules of $X$.

In this way approximations reveal granular structure of complex concepts. Thuse granularity of knowledge is inherently incorporated in the foundations of rough set theory.

\section{Dependencies and Granularity}

Another important issue in data analysis is discovering dependencies between attributes. Intuitively, a set of attributes $D$ depends totally on a set of attributes $C$, denoted $C \Rightarrow D$, if all values of attributes from $D$ are uniquely determined by values of attributes from $C$. In other words, $D$ depends totally on $C$, if there exists a functional dependency between values of $D$ and $C$.

We would need also a more general concept of dependency, called a partial dependency of attributes. Intuitively, the partial dependency means that only some values of $D$ are determined by values of $C$.

Formally dependency can be defined in the following way. Let $D$ and $C$ be subsets of $A$.

We will say that $D$ depends on $C$ in a degree $k(0 \leq k \leq 1)$, denoted $C \Rightarrow_{k} D$, if

$$
k=\gamma(C, D)=\frac{\operatorname{card}\left(P O S_{C}(D)\right)}{\operatorname{card} U},
$$

where

$$
\operatorname{POS}_{C}(D)=\bigcup_{X \in U / D} C_{*}(X),
$$

called a positive region of the partition $U / D$ with respect to $C$, is the set of all elements of $U$ that can be uniquely classified to blocks of the partition $U / D$, by means of $C$.

Obviously

$$
\gamma(C, D)=\sum_{X \in U / D} \frac{\operatorname{card}\left(C_{*}(X)\right)}{\operatorname{card} U} .
$$

If $k=1$ we say that $D$ depends totally on $C$, and if $k<1$, we say that $D$ depends partially (in a degree $k$ ) on $C$. 
The coefficient $k$ expresses the ratio of all elements of the universe, which can be properly classified to blocks of the partition $U / D$, employing attributes $C$ and will be called the degree of the dependency.

Obviously if $D$ depends totally on $C$ then $I_{C} \subseteq I_{D}$. That means that the partition generated by $C$ is finer than the partition generated by $D$.

Degree of dependency expresses to what extend granularity imposed by the set of attributes $D$ can be expressed in terms of elementary concepts associated with $C$.

The function $\gamma(C, D)$ can be regarded as a generalization of the rough inclusion function $\mu(X, Y)$, for it expresses to what degree partition generated by $C$, i.e., $U / C$ is included in the partition generated by $D$, i.e., $U / D$.

In other words, degree of dependency between $C$ and $D$ reveals to what degree granular structure imposed by $D$ can be expressed in terms of granular structure associated with $C$.

In fact approximations and dependencies are different sides of the same coin, and exhibit a relationship between two kinds of granular structures.

\section{Decision Rules}

With every dependency $C \Rightarrow_{k} D$ we can associate a set of decision rules, specifying decisions that should be taken when certain condition are satistied.

To express this idea more precisely we need a formal language associated with any information system $S=(U, A)$. The language is defined in a standard way and we omit detailed definition here, assuming that the reader is familiar with the construction (cf. Pawlak [15]).

By $\Phi, \Psi$ etc. we will denote logicals formulas built from attributes, attributevalues and logical connectives (and, or, not) in a standard way. We will denote by $\|\Phi\|_{S}$ the set of all object $x \in U$ satisfying $\Phi$ and refer to as the meaning of $\Phi$ in $S$.

The expression $\pi_{S}(\Phi)=\frac{\operatorname{card}\left(|| \Phi||_{S}\right)}{\operatorname{card}(U)}$ will denote the probability that the formula $\Phi$ is true in $S$.

A decision rule is an expression in the form "if...then...", written $\Phi \rightarrow \Psi$; $\Phi$ and $\Psi$ are refered to as condition and decision of the rule respectively.

The number $\operatorname{supp}_{S}(\Phi, \Psi)=\operatorname{card}\left(\|\Phi \wedge \Psi\|_{S}\right)$ will be called the support of the decision rule $\Phi \rightarrow \Psi$ in $S$ and the number

$$
\sigma_{S}(\Phi, \Psi)=\frac{\operatorname{supp}_{S}(\Phi, \Psi)}{\operatorname{card}(U)}
$$

will be reffered to as the strenght of the decision rule $\Phi \rightarrow \Psi$ in $S$.

If $\operatorname{supp}_{S}(\Phi, \Psi) \neq \Phi$ then the decision rule $\Phi \rightarrow \Psi$ will be called admissible in $S$.

In what follows we will consider admissible decision rules only.

A decision rule $\Phi \rightarrow \Psi$ is true in a degree $l(0 \leq l \leq 1)$ in $S$, if $\|\Phi\|_{S} \subseteq_{l}$ $\|\Psi\|_{S}$. 
With every decision rule $\Phi \rightarrow \Psi$ we associate a certainty factor

$$
\pi_{S}(\Psi \mid \Phi)=\frac{\operatorname{card}\left(\|\Phi \wedge \Psi\|_{S}\right)}{\operatorname{card}\left(\|\Phi\|_{S}\right)},
$$

which is the conditional probability that $\Psi$ is true in $S$ given $\Phi$ is true in $S$ with the probability $\pi_{S}(\Phi)$.

The certainty factor of a decision rule can be understood as the degree of truth of the decision rule or as the degree of inclusion of conditions in decisions of the decision rule. Besides, we will also need a coverage factor [24]

$$
\pi_{S}(\Phi \mid \Psi)=\frac{\operatorname{card}\left(\|\Phi \wedge \Psi\|_{S}\right)}{\operatorname{card}\left(\|\Psi\|_{S}\right)},
$$

which is the conditional probability that $\Phi$ is true in $S$ given $\Psi$ is true in $S$ with the probability $\pi_{S}(\Psi)$.

The coverage factor of a decision rule can be interpreted as the degree of truth of the inverse decision rule, or as the degree of the corresponding inclusion.

\section{Properties of Certainty and Coverage Factors}

Let $\Phi \rightarrow \Psi$ be a decision rule admissible in $S$. By $C(\Psi)$ we denote the set of all conditions of $\Psi$, such that if $\Phi^{\prime} \in C(\Psi)$ then $\Phi^{\prime} \rightarrow \Psi$ is admissible in $S$, and by $D(\Phi)$ we mean the set of all decisions of $\Phi$ such that if $\Psi^{\prime} \in D(\Phi)$ then $\Phi \rightarrow \Psi^{\prime}$ is admissible in $S$. Moreover we assume that all conditions in $C(\Psi)$ and all decisions in $D(\Phi)$ are pairwise mutually exclusive, i.e., if $\Phi^{\prime}, \Phi \in C(\Psi)$ then $\left\|\Phi^{\prime} \wedge \Phi\right\|_{S}=\emptyset$ and if $\Psi^{\prime}, \Psi \in D(\Phi)$ then $\left\|\Psi^{\prime} \wedge \Psi\right\|_{S}=\emptyset$. Then the following property holds:

$$
\begin{gathered}
\sum_{\Phi^{\prime} \in C(\Psi)} \pi_{S}\left(\Phi^{\prime} \mid \Psi\right)=1 \\
\sum_{\Psi^{\prime} \in D(\Phi)} \pi_{S}\left(\Psi^{\prime} \mid \Phi\right)=1 \\
\pi_{S}(\Psi)=\sum_{\Phi^{\prime} \in C(\Psi)} \pi_{S}\left(\Psi \mid \Phi^{\prime}\right) \cdot \pi_{S}\left(\Phi^{\prime}\right)=\sum_{\Phi^{\prime} \in C(\Psi)} \sigma_{S}\left(\Phi^{\prime}, \Psi\right) \\
\pi_{S}(\Phi)=\sum_{\Psi^{\prime} \in D(\Phi)} \pi_{S}\left(\Phi \mid \Psi^{\prime}\right) \cdot \pi_{S}\left(\Psi^{\prime}\right)=\sum_{\Psi^{\prime} \in D(\Phi)} \sigma_{S}\left(\Psi^{\prime}, \Phi\right) \\
\pi_{S}(\Phi \mid \Psi)=\frac{\pi_{S}(\Psi \mid \Phi) \cdot \pi_{S}(\Phi)}{\sum_{\Phi^{\prime} \in C(\Psi)} \pi_{S}\left(\Psi \mid \Phi^{\prime}\right) \cdot \pi_{S}\left(\Phi^{\prime}\right)}=\frac{\sigma_{S}(\Phi, \Psi)}{\pi_{S}(\Psi)} \\
\pi_{S}(\Psi \mid \Phi)=\frac{\pi_{S}(\Phi \mid \Psi) \cdot \pi_{S}(\Psi)}{\sum_{\Psi^{\prime} \in D(\Phi)} \pi_{S}\left(\Phi \mid \Psi^{\prime}\right) \cdot \pi_{S}\left(\Psi^{\prime}\right)}=\frac{\sigma_{S}(\Phi, \Psi)}{\pi_{S}(\Phi)}
\end{gathered}
$$


Formulas 3) and 4) are the total probability theorems, whereas formulas 5) and 6) are the Bayes' theorems. The relationship between truth of implications and the Bayes' theorem first was observed by Łukasiewicz [4, 13] (see also [1]). The meaning of Bayes' theorem in this case differs from that postulated in statistical inference, where we assume that prior probability about some parameters without knowledge about the data is given. The posterior probability is computed next, which tells us what can be said about prior probability in view of the data.

In the rough set approach the meaning of Bayes' theorem is unlike. It reveals some relationships between decision rules, without referring to prior and posterior probabilities. Instead, the proposed approach connects the total probability theorem and the Bayes, theorem with the strength of decision rules, giving a very simple way of computing the certainty and the coverage factors.

Thus, the proposed approach can be seen as a new model for Bayes' theorem, which offers a new approach to data analysis, in particular, to inverse decision rules and to compute their certainty factors, which can be used to explain decisions in terms of conditions.

\section{Rough Modus Ponens and Rough Modus Tollens}

The above considerations can be seen as a generalization of modus ponens and modus tollens inference rules.

Modus ponens inference rule says that:

$$
\begin{aligned}
& \text { if } \Phi \rightarrow \Psi \text { is true } \\
& \text { and } \Phi \quad \text { is true } \\
& \hline \text { then } \Psi \text { is true }
\end{aligned}
$$

This rule can be generalized as rough modus ponens as follows. For any $\Phi \rightarrow \Psi$ we have

$$
\begin{aligned}
& \text { if } \Phi \rightarrow \Psi \text { is true with the probability } \\
& \text { and } \Phi \quad \begin{aligned}
& \pi_{S}(\Psi \mid \Phi) \\
& \text { is true with the probability } \\
& \pi_{S}(\Phi) \\
& \hline \text { then } \Psi \text { is true with the probability } \\
& \pi_{S}(\Psi)=\sum_{\Phi^{\prime} \in C(\Psi)} \pi_{S}\left(\Psi \mid \Phi^{\prime}\right) \cdot \pi_{S}\left(\Phi^{\prime}\right)=\sum_{\Phi^{\prime} \in C(\Psi)} \sigma_{S}\left(\Phi^{\prime}, \Psi\right)
\end{aligned}
\end{aligned}
$$

Similarly, modus tollens inference rule

$$
\begin{aligned}
& \text { if } \Phi \rightarrow \Psi \text { is true } \\
& \frac{\text { and } \sim \Psi \text { is true }}{\text { then } \sim \Phi}
\end{aligned}
$$


can be generalized as rough modus tollens as follows. For any $\Phi \rightarrow \Psi$ we have

$$
\begin{array}{ll}
\text { if } \Phi \rightarrow \Psi & \text { is true with the probability } \\
& \pi_{S}(\Psi \mid \Phi) \\
\text { and } & \Psi \text { is true with the probability } \\
& \pi_{S}(\Psi) \\
\hline \text { then } \Phi & \text { is true with the probability } \\
& \pi_{S}(\Phi)=\sum_{\Psi^{\prime} \in D(\Phi)} \pi_{S}\left(\Phi \mid \Psi^{\prime}\right) \cdot \pi_{S}\left(\Psi^{\prime}\right)=\sum_{\Psi^{\prime} \in D(\Phi)} \sigma_{S}\left(\Psi^{\prime}, \Phi\right)
\end{array}
$$

Due to the Bayes' theorem (5) and symmetry of strength of decision rules we get

$$
\pi_{S}(\Phi)=\sum_{\Psi^{\prime} \in D(\Phi)} \sigma_{S}\left(\Phi, \Psi^{\prime}\right)
$$

The generalizations of both inference rules consist in replacing logical values of truth and falsehood with their probabilities in accordance with the total probability theorem (3),(4) and the Bayes' theorem (5),(6).

\section{Conclusions}

Granularity of knowledge, information, measurements, computations etc., seems to be an intrinsic feature of our thinking and can be considered as a manifestation of an old antinomy associated with continuos-discrete paradigm.

Rough set philosophy hinges on the granularity of data, which is used to build up all its basic concepts, like approximations, dependencies, reduction etc.

Particularly interesting in this approach seems to be the relationship between partial truth, rough mereology, Łukasiewicz's many-valued logic and Bayes' theorem.

\section{Acknowledgments}

Thanks are due to Professor Andrzej Skowron for his critical remarks and helpful comments.

\section{References}

1. Adams E. W. (1975) The Logic of Conditionals, an Application of Probability to Deductive Logic. D. Reidel Publishing Company, Dordrecht, Boston

2. P. Apostoli, A. Kanda, Parts of the continuum: towards a modern ontology of science, (to appear), 1999

3. Black M. (1952) The Identity of Indiscernibles, Mind, 61

4. Borkowski L. (Ed.) (1970) Jan Łukasiewicz - Selected Works, North Holland Publishing Company, Amsterdam, London, Polish Scientific Publushers, Warszawa 
5. Cattaneo G. (1993) Fuzzy Quantum Logic: The Logic of Unsharp Quantum Mechanics. Int. Journal of Theoretical Physics 32:1709-1734

6. Cattaneo G. (1996) Mathematical Foundations of Roughness and Fuzziness. In: Tsumoto S. at al (Eds.) The fourth International Workshop on Rough Sets, Fuzzy Sets and Machine Discovery, Proceedings, The University of Tokyo 241247

7. Chattebrjee A. (1994) Understanding vagueness, pragati publications. Dehli

8. Dubois D., Prade H. (1999) Foreword. In: Pawlak Z. Rough Sets - Theoretical Aspect of Reasoning about Data, Kluwer Academic Publishers, Dordrecht, Boston , London

9. Forrest P. (1997) Identity of Indiscernibilities. Stanford Encyclopedia of Philosophy

10. French S. (1998) Quantum Physics and the Identity of Indiscernibles. British Journal of the Philosophy of Sciences 39

11. Krawiec K., Słowiński R., Vanderpooten D. (1996) Construction of Rough Classifiers Based on Application of Similarity Relation. In: Proceedings of the Fourth International Workshop on Rough Sets, Fuzzy Sets and Machine Discovery, November 6-8, Tokyo, Japan, 23-30

12. Leśniewski S. (1992) Foundations of the General Theory of Sets. In: Surma, Srzednicki, Barnett, Riskkey (Eds) Stanislaw Leśniweski Collected Works, Kluwer Academic Publishers, Dordrecht, Boston, London, 128-173

13. Łukasiewicz J. (1913) Die logishen Grundlagen der Wahrscheinichkeitsrechnung, Krakow

14. Parker-Rhodes A. F. (1981) The Theory of Indistinguishables. D. Reidel Publishing Company, Dordrecht, Boston, London

15. Pawlak, Z. (1998) Granurality of Knowledge, Indiscernibility and Rough Sets. In: IEEE International Conference on Granulationary Computing, May 5-9, Anchorage, Alaska, 100-103

16. Polkowski L., Skowron A. (1997) Towards Adaptative Calculus of Granules. Manuscript

17. Polkowski L., Skowron A. (1994) Rough Mereology. In: Proc. of the Symphosium on Methodologies for Intelligent Systems, Charlotte, N.C., Lecture Notes in Artificial Intelligence 869, Springer Verlag, Berlin, 85-94

18. Polkowski L., Skowron A. (1996) Rough Mereology: A new Paradigm for Approximate Reasoning. Journ. of Approximate Reasoning 15(4):333-365

19. Polkowski L., Skowron A. (Eds.) (1998) Rough Sets in Knowledge Discovery. Physica-Verlag Vol. 1, 2

20. Skowron A., Stepaniuk J. (1997) Information Granulation - a Rough Set Approach. Manuscrript

21. Skowron A., Stepaniuk J. (1996) Tolerance Approximation Spaces. Fundamenta Informaticae 27:245-253

22. Sorensen R. (1997) Vagueness. Stanford Encyclopedia of Philosophy

23. Suppes P. (1972) Some Open Problems in the Philosophy of Space and Time. Synthese 24:298-316

24. Tsumoto S. (1998) Modelling Medical Diagnostic Rules Based on Rough Sets. In: Polkowski L., Skowron A. (Eds.) Rough Sets and Current Trends in Computing, Lecture Notes in Artificial Intelligence 1424 Springer, First International Conference, RSCTC'98, Warsaw, Poland, June, Proceedings, 475-482

25. Williamson T. (1990) Identity and Discrimination. Blackwell 
26. Yao Y.Y., Wong S.K.M. (1995) Generalization of Rough Sets using Relationships between Attribute Values. In: Proceedings of the Second Annual Joint Conference on Information Sciences, Wrightsville Beach, N.C. USA, September 28 - October 1, 245-253

27. Zadeh L. (1994) Fuzzy Graphs, Rough Sets and Information Granularity. In: Proc. Third Int. Workshop on Rough Sets and Soft Computing, Nov. 10-12, San Jose

28. Zadeh L. (1996) The Key Rules of Information Granulation and Fuzzy Logic in Human Reasoning, Concept Formulation and Computing with Words. In: Proc. FUZZ-96: Fifth IEEE International Conference on Fuzzy Systems, September 8-11, New Orleans

29. Zadeh L. (1996) Information Granulation, Fuzzy Logic and Rough Sets. In: Proc. of the Fourth Int. Workshop on Rough Sets, and Machine Discovery, November 6-8, Tokyo

30. Ziarko W. (1993) Variable Precison Rough Set Model. Journal of Computer and System Sciences 46/1:39-59 